\title{
On the Development of a Vibration Energy Harvesting Mechanism and the Influence of the Number of Wire Turns
}

\author{
Wiwiek Hendrowati ${ }^{1}$, Bambang Daryanto W. ${ }^{1}$, and I Nyoman Sutantra ${ }^{1}$
}

\begin{abstract}
This article deals with a research activity to design and to build a vibration energy harvesting (VEH) mechanism based on an electromagnetic method, where the energy source is from translational-harmonic vibration. In the developed VEH mechanism, a magnetic mass moves linearly back and forth within an electrical coil made from conductive-wire windings. In accordance to oscillating mass motion of a particular amplitude and frequency, the voltage which can be harvested is then measured and analyzed. The choice of an electromagnetic method stemmed from the availability of materials to construct the VEH mechanism. In the VEH mechanism, a mass size, a wire diameter and material, a coil length, and a vibration amplitude and frequency were considered constant, while the number of wire turns was varied. The constructed VEH was tested for $1,000,1,500,2,000,2,500$, and 3,000wire turns. The voltage density being harvested was recorded as $0.2820,0.3715$, $0.5695,0.7343$ and 0.9300 volt $/ \mathrm{cm}^{3}$, respectively.
\end{abstract}

Keywords $\longrightarrow$ vibration energy harvesting, electro-magnetic method, translational vibration, wire turns

\section{INTRODUCTION}

$\mathrm{T}$ here are many activities that are related to the advancement of technology; activities in industry, transportation, communication, and domestic affairs. The utilization of technological development is intended to support or to ease human jobs, so that he/she may complete his/her tasks in effective and efficient manner. Technological development itself requires a support of the availability of energy resources to drive equipment, where one of resources is the electrical energy. The required energy may come from another source which is converted into an electrical energy. One recognizes macro electrical energy conversion (from sources such as water, wind, geothermal), which yields an electrical energy of a big scale. Besides that, there is micro-scale electrical energy conversion, which usually uses surroundings energy (such as vibration, temperature difference, light) to be converted into an electrical energy.

Among sources of micro-scale electrical energy conversion, vibration or motion is an interesting source. Anything that moves or any equipment that operates (whether with a high or a low velocity) will vibrate, and yields oscillating motion that happens for a rather long period. Whilst, so far it is detected that vibration or motion does not receive enough attention it deserves when it comes to energy conversion. Due to the aforementioned background the research to harvest energy from vibration was conducted.

${ }^{1}$ Wiwiek Hendrowati, Bambang Daryanto W., and I Nyoman Sutantra are with Department of Mechanical Engineering, FTI, Institut Teknologi Sepuluh Nopember, Surabaya, 60111, Indonesia. E-mail: wiwiek@me.its.ac.id.
In vibration, the energy comes from a relative displacement between a mass and a system frame, because there is flexibility between them. The relative displacement is in the form of oscillated motion, where the incurred motion indicates that there is a velocity and an acceleration of motion which change both their value and direction. The vibration itself is associated with a kinetic and a potential energy. And in turns, the vibration energy can be converted into an electrical energy through electrical field (electrostatic), magnetic field (electromagnet), or piezoelectric material strain [1]. It must be brought into attention that there is dissipation of energy due to damping within the system, because of the existence of friction and a damper in the system.

Some researcher and or publications have reported energy harvesting from a vibration source to an electrical energy, using a piezoelectric method. The method was applied to industrial equipment and to human activities to harvest the intended energy $[2,3]$. The varying (with time) displacement was accomplished in the form of cantilever beam deflection [4]. Beside piezoelectric materials, magnetostrictive materials were also used to harvest energy from vibration [5]. Implementation on a military vehicle, where the harvested energy was from vibration of a vehicle chassis and vibration due to crankshaft rotation [6].

Design and manufacture of a micro generator based on an electromagnetic method is discussed in [7], where its standard analysis is found in [8]. Harvesting energy from a rotating wheel of varying speed was conducted in [9], while scavenging low frequency of non-periodic vibration was done in [10]. A parametrical research of an electromagnetic micro generator is re-ported in [11], where the number and the size of wire turns to build a circular-shape coil (instead of a longitudinal-shape coil) were varied. Using computer simulation, a research in [12] investigated the effect of a magnetic field scale and coil parameters on micro generator performances.

Based on the referred background, this article concerns a research activity to design and to build a vibration energy harvesting (VEH) mechanism according to an electromagnetic method, to investigate the energy that can be harvested from a translational vibration source. The electromagnetic method becomes a research focus due to the fact that required materials to build a VEH mechanism are relatively easy to find in the market (in Indonesia). In the developed mechanism, a magnetic mass moves linearly back and forth within an electrical coil made from conductive-wire windings. Mass motion represents oscillatory motion with a particular amplitude (stroke) and frequency. From vibration which happens, the motion energy is then 'captured' by a coil and therefore produces an electrical energy. 


\section{THEORETICAL BACKGROUND}

\section{A. Mechanical Vibration}

Fig. 1 schematically shows a mass $m$ which receives excitation in the form of base motion $y(t)$. According to the model, a motion equation of a mass response $x(t)$ can be expressed as:

$m \ddot{x}+c(\dot{x}-\dot{y})+k(x-y)=0$

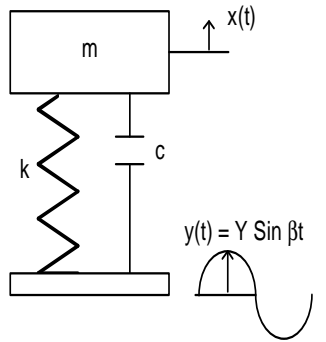

Fig. 1. Mass motion due to base excitation

If $y(t)$ is harmonic motion of an amplitude $Y$ and a frequency $\beta$, then steady-state motion of the mass can be expressed as:

$x(t)=\frac{Y \sqrt{k^{2}+(c \beta)^{2}}}{\sqrt{\left(k-m \beta^{2}\right)^{2}+(c \beta)^{2}}} \sin \left(\beta t-\phi_{1}-\alpha\right)$

where

$\phi_{1}=\tan ^{-1}\left(\frac{c \beta}{k-m \beta^{2}}\right)$

$\alpha=\tan ^{-1}-(c \beta / k)$

Equation (2) indicates that, if excitation $y(t)$ is oscillatory-harmonic motion then a response $x(t)$ is harmonic vibration also. In this reported research, harmonic excitation $y(t)$ comesfrom a disc which rotates eccentrically.

\section{B. Electromagnet}

If the displacement (position) of a magnetic mass changes with time and the mass moves within or surrounded by a coil which is charged by electrical current, then magnetic field is excited or inducted. (See Fig. 2.)

According to Faraday's rule, for varied magnetic field or flux, within a coil will be excited an emf (electromotive force) of:

$e=-N \frac{d \phi}{d t}=-N \frac{d(B \cdot A)}{d t}$

where

$B=$ flux density $\left(\mathrm{Wb} / \mathrm{m}^{2}\right)$

$A=$ cross-section area which cut emf

$N=$ number of windings in coil

$\phi=$ flux value (varies with time)

If flux density and cross-section area are constant, Equation (5) becomes:

$e=-N . B . A . f$

Equation (6) shows that the resulted energy depends on number of turns, magnitude of magnetic flux, crosssection area which cut emf, and frequency of magnet motion.

\section{Magnetic Field of Solenoid}

A solenoid is a coil consists of several (many) coaxially circular loops of the same radius, and is charged with the same electrical flow. (See Fig. 3.)

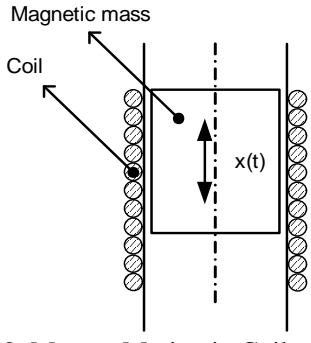

Fig. 2. Magnet Motion in Coil

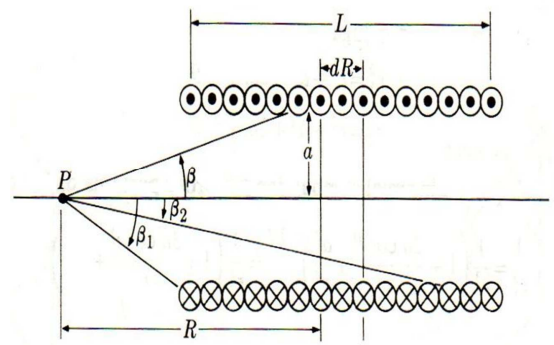

Fig. 3. Flux density of point $P$ located on axis of solenoid

If $L$ denotes a length and $N$ denotes a number of wire windings, then $N / L$ is the number of windings per unit length. The number of wire windings for $d R$ is $(N / L) d R$. And, flux density can be calculated as:

$B=\frac{\mu_{0} I N}{2 L} \int_{\beta_{1}}^{\beta_{2}}-\sin \beta d \beta=\frac{\mu_{0} I N}{2 L}\left(\cos \beta_{2}-\cos \beta_{1}\right)$

where

$\mu_{o}$ : permeability of free space $=4 \pi \times 10^{-7} \mathrm{H} / \mathrm{m}$

I: electric current

For the value of $\beta_{1} \approx \pi$ and $\beta_{2} \approx 0$, Equation (7) gives

$B=\frac{\mu_{0} I N}{L}$

and for a point located at either end of the coil $\left(\beta_{1} \approx \pi / 2\right.$ and $\beta_{2} \approx 0$, or $\beta_{1} \approx \pi$ and $\beta_{2} \approx \pi / 2$ ) Equation (8) becomes

$B=\frac{\mu_{0} I N}{2 L}$

\section{Energy Harvesting from Vibration}

Fig. 4 shows a basic design/process of energy harvesting from vibration, where the main section consists of a mechanical system and an electrical system. On the mechanical section there is a magnetic mass which periodically moves oscillatory, following/according to motion of a given amplitude and frequency. Whereas in the electrical section there is a coil made of conductivewire windings which physically surround mass motion. Mass motion within a coil will excite electron motion in conductor wire, and hence electrical current is inducted in the coil. In other words, vibration motion of a mass can become a source for producing an electrical energy.

The excited electrical current in the coil is in the form of alternating current, which is still pulsating. The current is then flowed through four diodes which are arranged to form a bridge, aimed to make the current unidirectional. Output of the diodes bridge is direct current, and hence may be stored in a battery. The function of a capacitor in an electrical part is to eliminate fluctuation of current or to smooth current, and to serve as a temporary storage of current. In turns, the harvested electrical energy which is stored in a battery (from mass vibration) can be used by or flowed to necessitated loads. 


\section{METHOD}

The main objective of the research is to create a VEH mechanism that can function properly. After the intended mechanism is built, the mechanism is tested in order to create a 'workable' VEH from which the electrical energy is generated.

The objective of the research is realized through several steps:

1. comprehensive literature study

2. design, manufacture and test of a VEH mechanism

3. experimental study of a VEH mechanism of different number of wire turns, and

4. analyses of experiment results.

Schematically, the design of a prototype of a VEH mechanism based on an electromagnetic method for a translational-harmonic vibration source is as shown by Fig. 5.

In short description, the primary works of a VEH mechanism is designated as follows: Translational oscillation comes from a pushrod connected to an eccentric disc, where a DC motor serves as a prime mover to rotate the disc. The other end of a push-rod is connected to a mass. According to the mechanism, the mass moves back and forth harmonically. The spring is intended to make sure that the wheel of a pushrod is always in contact with the outer surface of the eccentric disc. The magnetic mass oscillates within an electrical coil made from conductive-wire windings. The linear motion of the mass emulates vibration from which the energy is harvested. Due to the harmonic motion of a magnetic mass, electrical current is inducted in the coil. Then, the electrical current flow is stored by an energy storage device (a storage battery). Within this context, the electrical energy represents the ensuing energy that can be harvested from the vibration.

The prototype of a VEH mechanism is as shown by Fig.6. The prototype consists of light components/ materials, but is stable under given vibration.

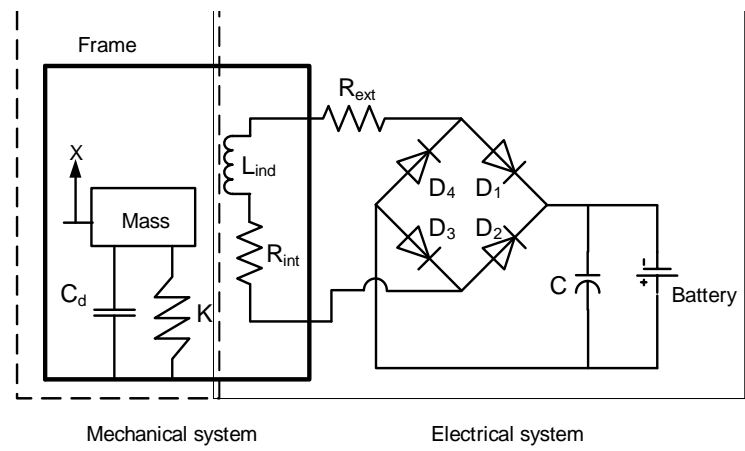

Fig. 4. Diagram of mechanical and electrical system

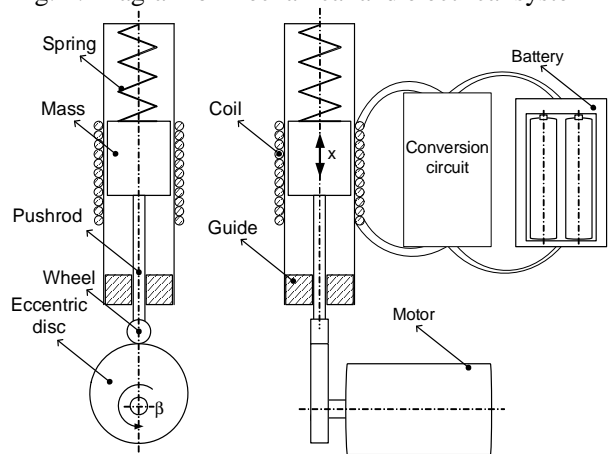

Fig. 5. Diagram of VEH mechanism based on electromagnetic method
The main parts of the mechanism are the magnetic mass and the coil. The reported research uses a mass of $0.013 \mathrm{~kg}$ (15 mm diameter, $12 \mathrm{~mm}$ long), with 3500 Gauss flux density. The coil (where the number of windings is varied) consists of many layers, is made from $0.1 \mathrm{~mm}$ wire diameter, has the length of $25 \mathrm{~mm}$ and the inside diameter of $18 \mathrm{~mm}$. The stroke of a pushrod is $20 \mathrm{~mm}$, and the frequency of mass motion is $14.5833 \mathrm{~Hz}$. For the VEH mechanism, from which data are collected, the length of the coil is designed to cover up to (approximately) 0.7 of the mass length, where the mass moves back and forth. In other words, due to back-andforth motion, the mass is displaced up to about $3.5 \mathrm{~mm}$ of its length 'protrudes' outside the coil.

The manufactured VEH mechanism is then tested in order to build a VEH prototype which functions properly, i.e. from the operation of the mechanism electrical energy can be harvested. The energy represents energy harvesting from a source of translationalharmonic vibration. For each mechanism setting (operation), the resulted voltages and amperes are measured, and hence the amount of the harvested electrical energy can be known.

On the 'workable' VEH the experiment is con-ducted with the number of turns of 1000, 1500, 2000, 2500, and 3000 , to study the influence of the number of wire turns on the harvested/generated energy. To make the coil of (the same) 25-mm long, wire turns are wound in many layers. For each variation of the windings, results given by the VEH mechanism are shown as graphics of the induced voltages and amperes.

\section{RESULT AND ANALYSIS}

The recorded data come from the VEH proto-type which is developed in the reported research. The developed prototype has been tested, and the mechanism functions properly.

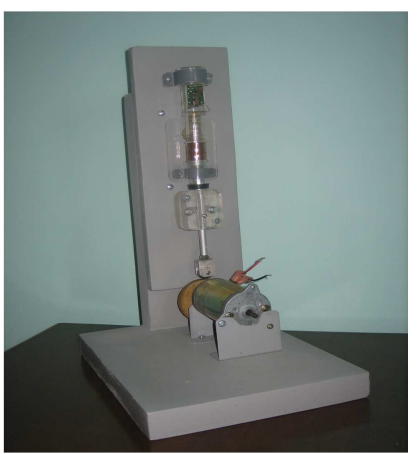

Fig. 6. Photograph of VEH mechanism based on electromagnetic method

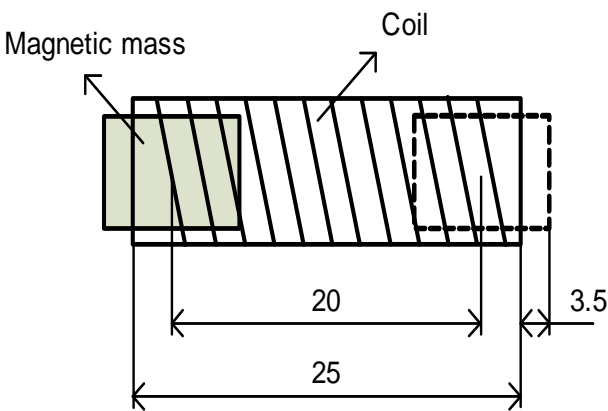

Fig. 7. Coil length and mass motion 

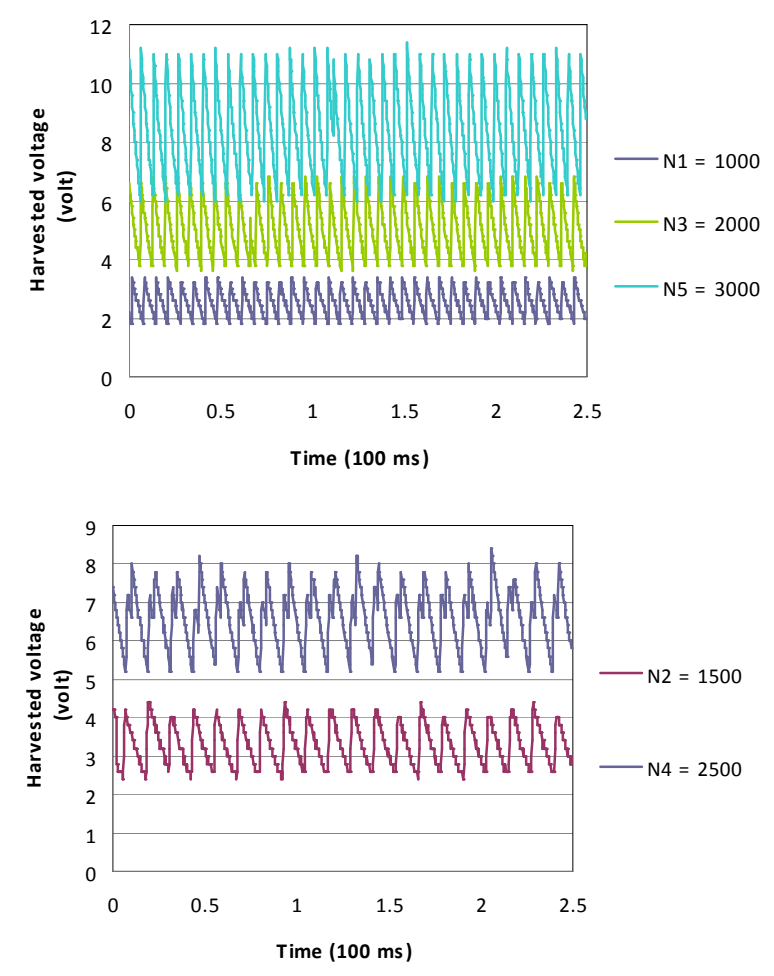

Fig. 8. Voltage generated by VEH mechanism

The study is then conducted on the VEH mechanism for different number of wire turns with the excitation of translational-harmonic vibration. The data are taken 5 times for every experiment setting (for every number of wire turns). The vibration comes from a mass which moves back and forth due to the $875 \mathrm{rpm}$ rotation of a DC motor. The frequency and amplitude (of $10 \mathrm{~mm}$ ) of vibration are used throughout the experiment. The results of the experiment are represented by voltages and current generated by the VEH mechanism, which are recorded by an oscilloscope.

Fig. 8 typically shows voltages which can be produced by the VEH mechanism, for the wire turns of 1000. For 1000 wire windings the generated voltage $\mathrm{rms}$ is 2.55 volts. Accompanied by a relatively same graph, the VEH mechanism with 1500 turns generates voltage rms of 3.36 volts; for 2000 turns the generated voltage $\mathrm{rms}$ is 5.15 volts; for 2500 turns the generated voltage $\mathrm{rms}$ is 6.64 volts; and for 3000 turns the generated voltage rms is 8.41 volts. Recorded voltages indicate that, for a particular coil length, a higher number of wire turns generate a higher voltage and a wider voltage range.

The electric current is measured by adding a 100-Ohm resistance to the oscilloscope. Fig.9 typically shows current produced by the VEH mechanism, for the wire turns of 1000 . For 1000 wire windings the current rms is 0.51 milli-amperes. Accompanied by a relatively same graph, the VEH mechanism with 1500 turns generates rms of 1.12 milli-amperes; for 2000 turns the rms is 1.53 milliamperes; for 2500 turns the rms is 2.31 milliamperes; and for 3000 turns the rms is 2.25 milliamperes. The generated energy/power by the developed VEH mechanism can be then calculated from the re-corded voltage and ampere data.They are $1.3005 \mathrm{mVA}, 3.7632 \mathrm{mVA}$, $7.8795 \mathrm{mVA}, 15.3384 \mathrm{mVA}, 18.9225 \mathrm{mVA}$ for 1000 , 1500, 2000, 2500, 3000 wire turns, respectively.
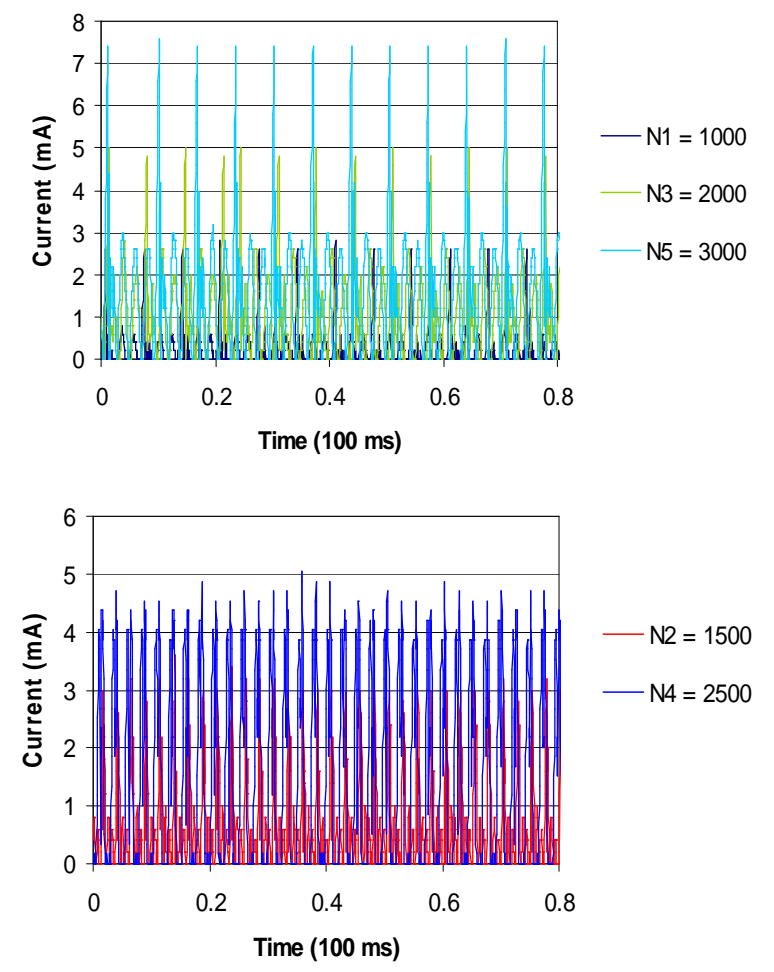

Fig. 9. Ampere generated by VEH mechanism

With regard to the $\mathrm{VEH}$ mechanism size, the (approximate) generated voltage densities and energy densities as results of the experiment are tabulated in Table 1 . The density is calculated by dividing the generated voltage and energy by the effective volume of the prototype. The aforementioned effective volume is the volume of the coil cartridge, which is equal to the volume of a cylinder of $32 \mathrm{~mm}$ long and $18 \mathrm{~mm}$ diameter.

Fig.10 shows the influence of a number of wire turns on the voltage density produced by the VEH mechanism. It can be seen from Fig.10 that the volt-age density increases with the addition of wire turns. This fact agrees with a research reported in [9], using a different mechanism design. The increase in wire wind-ings means that the conductive wire is longer. Where, the conductive wire contains electrons that change into an electrical current under the influence of magnetic field. This fact agrees with Equation 6, which indicates that the emf produced by a VEH mechanism will increase with the increase of the number of wire windings in a coil. It also agrees with a research (using a different base-design mechanism) reported in [9], where higher number of wire turns yielded higher voltages. The corresponding generated energy/power density is shown in Fig. 11 of this article.

The fluctuation in the recorded voltage and current, in part, is due to the use of the spring to ensure that the pushrod wheel and the outer surface of the eccentric disc are always in contact. When the mass motion is up, the VEH mechanism is against the stiffness of the spring. When the motion is down, the spring acts as a pusher.

For the record, the aim of the research in [13] is to study the influence of the amplitude and frequency of the excitation on the harvested energy. The research reported that an increase of the amplitude and frequency yielded bigger harvested energy. 


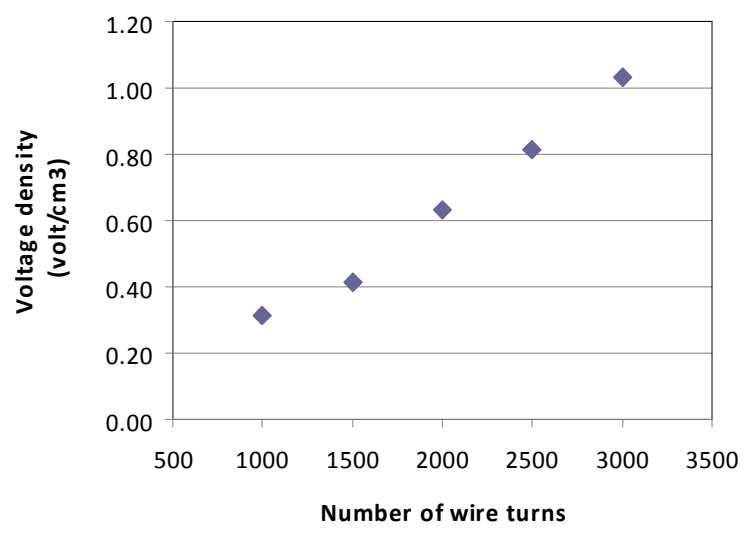

Fig. 10. Voltage density vs. number of wireturns

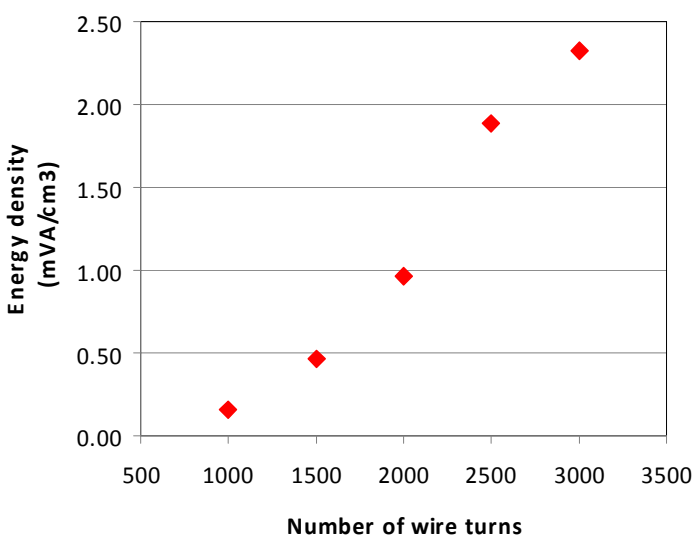

Fig. 11. Energy density vs. number of wire turns

TABLE 1.

GENERATED VOLTAGE DENSITY $\left(10^{-3} \mathrm{x}\right.$ V/CM $\left.{ }^{3}\right)$ AND ENERGY DENSITY $\left(10^{-3} \mathrm{X} \mathrm{MVA} / \mathrm{CM}^{3}\right)$

\begin{tabular}{lccccc}
\hline Number of Wire Turns & 1000 & 1500 & 2000 & 2500 & 3000 \\
\hline Voltage Density & 0.3133 & 0.4128 & 0.6328 & 0.8158 & 1.0333 \\
\hline Energy Density & 0.1598 & 0.4624 & 0.9681 & 1.8846 & 2.3250 \\
\hline
\end{tabular}

\section{CONCLUSION}

The research activity has created a prototype of VEH mechanism. The study has been conducted on the mechanism using coils consist of different number of wire turns, while other parameters of the mechanism remain the same. The reported experiment attests the influence of the number of wire windings to be used to build a coil in the VEH mechanism, where (for a particular coil length) a higher number of wire windings generate a higher energy. Fig. 10 and 11 shows the relation between the number of wire turns and the generated voltage and energy is close to linear.

The reported research is an example of a real activity to contribute to the effort of harvesting energy from a vibration source. The research is intended to be a trigger to the development of a VEH mechanism for a microgenerator (in Indonesia), including the mechanism applicable implementation.

\section{REFERENCES}

[1] Torres, O. Erick, 2005, "Energy-harvesting chips and the quest for everlasting life", Power Management Design Line, Texas Instru-ments, Houston.

[2] Nuffer, Jurgen, T. Bein, 2006, "Application of piezoelectric materials in transportation industry", Proc. Symposium on Innovative Solutions for Advancement of Transport Industry, San Sebastian, Spain.

[3] Kymissis, John, C. Kendall, J. Paradiso, N. Gershenfeld, 2008, "Parasitic power harvesting in shoes", Proc. $2^{\text {nd }}$ Intl. Symposium on Wearable Computing, Pittsburgh, PA.

[4] Umeda, M., K. Nakamura, S. Ueha, 1996, "Analysis of transformation of mechanical impact energy to electric energy using piezoelectric vibrator", Japan J. Applied Physics, Vol. 3B, pp. 3267-3273.
[5] Wang, Lei, F.G. Yuan, 2007, "Energy harvesting by Magnetostrictive Material (MsM) for powering wireless sensors in SHM", Dept. of Mech. and Aerospace Eng., North Carolina State Univ., Raleigh, NC.

[6] Daqaq, F. Moh., J. Wagner, M. Letherwood, C. Stabler, 2009 "Supplemental vehicle power through innovative energy harvesting", Automotive Research Center, Ann Arbor, MI.

[7] Pan, C.T., and T.T. Wu, 2007, "Development of rotary electromagnetic microgenerator", Journal of Micromechanics and Microengineering (UK), Vol. 17, pp. 120-128.

[8] Beeby, Steve P., M.J. Tudor, R.N. Torah, E. Koukharenko, S. Roberts, T. O'Donnell, and S. Roy, 2006, "Macro and micro scale electromagnetic kinetic energy harvesting generators", Design, Test, Integration and Packaging of MEMS and MOEMS, Stresa, Italy.

[9] Y-J Wang, C-D. Chen, C-K. Sung, 2010, "Design of a frequency adjusting device for harvesting energy from a rotating wheel", Journal Sensors and Actuators A: Physical, Vol. 159, pp. 196203.

[10] T. Galchev, H. Kim, K. Najafi, 2009, "A parametric frequency increased power generator for scavenging low frequency ambient vibrations", Journal Procedia Chemistry, pp. 14391442.

[11] Beeby, Steve P., M.J. Tudor, R.N. Torah, E. Koukharenko, S. Roberts, T. O’Donnell, S. Roy, 2007, "A microelectronic generator for vibration energy harvesting", Journal Micromechanics and Micro engineering (UK), Vol. 17, pp. 1257-1265.

[12] O'Donnell, Terence, C. Saha, S.P. Beeby, M.J. Tudor, 2006, "Scaling effects for electromagnetic vibration power generator", Design, Test, Integration and Packaging of MEMS and MOEMS, Stresa, Italy.

[13] Daryanto, Bambang, H.L. Guntur, 2010, "Rancang bangun mekanisme vibration energy harvesting dan analisa energi yang dihasilkan untuk amplitudo dan frekuensi getaran yang bervariasi", Laporan Penelitian, LPPM-ITS, Surabaya. 
